\title{
INNOVACIÓN SOCIAL, INNOVACIÓN SMART: NUEVAS RESPUESTAS FRENTE A LOS DESAFÍOS DE LA HUMANIDAD
}

\author{
SOCIAL INNOVATION, SMART INNOVATION: \\ NEW RESPONSES TO THE CHALLENGES OF HUMANITY
}

\author{
Blanca Herrero de Egaña \\ Universidad Pontificia de Comillas, Madrid \\ blanca.hmc@benefactor.es
}

\begin{abstract}
Resumen: El presente artículo propone explicar cómo la innovación social, entendida como «formas nuevas o novedosas que tiene la sociedad para hacer frente a los retos sociales relevantes, que son más eficaces, eficientes y sostenibles o que generan mayor impacto que las precedentes y que contribuyen a hacerla más fuerte y articulada» (Herrero de Egaña, 2018a) y, concretamente, la innovación social smart o innovación inteligente, puede ser la respuesta local a los desafíos de la humanidad. La crisis del estado de bienestar, el aumento y la diversificación de necesidades y desafíos sociales y la ineficacia de los modelos preexistentes invitan a buscar soluciones novedosas, con mayor impacto y más sostenibles, en las que se creen y se refuercen nuevos modelos y tipos de relación. En esta investigación se explora y desarrolla una de las líneas de investigación que quedaron abiertas tras el trabajo de investigación que, durante los años 2013-2018 he realizado para entender la innovación social en España a través de la Teoría Fundamentada (Glaser \& Strauss, 1967). Gracias a la recogida, codificación, análisis y comparación constante de un corpus de más de 3000 entradas y de más 50 casos de estudio de innovación social en España, he podido aportar una definición propia de innovación social, una innovación inteligente, cuyo impacto a nivel micro, meso o macro podría medirse y cuantificarse. El estudio empírico, comparado y anclado en los datos, a partir de casos de estudio sobre innovación social smart en España, permite evidenciar como ésta contribuye decisivamente a hacer frente a los desafíos globales que enfrenta la humanidad para un mundo mejor, más justo y más humano.
\end{abstract}

Palabras clave: Innovación social, innovación smart, retos sociales relevantes, estudio de casos, Teoría Fundamentada, análisis cualitativo.

\begin{abstract}
This article explains how social innovation, understood as «new or novel ways that society has to deal with relevant social challenges, which are more effective, efficient and sustainable or generate greater impact than the preceding ones and contribute to making it stronger and more articulate» (Herrero de Egaña, 2018) and, in particular, social innovation or smart innovation can be the local response to the global challenges of humanity. The crisis of the welfare state, the increasing and the diversifying needs and social challenges and the ineffectiveness of existing models invite you to find innovative solutions, with greater impact and more sustainable, in which new models and types of relationship are created and reinforced. This paper explores and develops one of the research lines that remained open after the research work I have conducted during the years 2013-2018 in order to understand social innovation in Spain through Grounded Theory (Glaser \& Strauss, 1967). Thanks to the collection, analysis coding and constant comparison of a corpus of more than 3,000 entries and more than 50 case studies of Spanish social innovation, I have been able to provide an own definition of social innovation, a smart innovation, whose impact at the micro, meso or macro level could be measured and quantified. The empirical study, compared and anchored in the data of cases of smart social innovation in Spain, allows to evidence how this one contributes decisively to tackle the global challenges humanity faces for a better, fairer and more humane world. The empirical study, compared and anchored in the data of cases of smart social innovation in Spain, allows to evidence how this one contributes decisively to tackle the global challenges humanity faces for a better, fairer and more humane world.
\end{abstract}

Keywords: Social innovation, smart innovation, relevant social challenges, case studies, Grounded Theory, qualitative analysis. 


\section{Introducción}

El propósito de este apartado introductorio es, en primer lugar, describir el contexto en que se desarrolla esta investigación y que justifica la pertinencia, oportunidad e importancia de la misma. Así mismo, se hace necesaria una breve referencia a la metodología utilizada para desarrollar la presente investigación, la Grounded Theory (en adelante, Teoría Fundamentada). En este apartado se explicará cómo ha sido aplicada y adaptada a las características de este trabajo y su contexto.

En tercer lugar, se hace referencia a varios conceptos utilizados en el artículo, como innovación social o retos sociales relevantes (RSR), y su definición en el contexto de este trabajo.

Por último, se hace referencia al corpus de la investigación y, en concreto, a los criterios de selección de los cincuenta casos de innovación social española a partir de los cuales se explica cómo la innovación social en general y la innovación smart, en particular, contribuyen de forma significativa a los desafíos sociales de la humanidad.

\section{La selección del tema de estudio: contexto y justificación}

La innovación social, entendida como una nueva forma de satisfacer necesidades sociales no resueltas, paradójicamente no es algo nuevo. Desde siempre han existido reacciones colectivas y novedosas ante las necesidades sociales no cubiertas, como por ejemplo los montes de piedad, los gremios, los ateneos obreros, o las cooperativas, entre otros.

Sin embargo, es innegable el creciente protagonismo que la innovación social está cobrando en los últimos años.

Varios son los factores que han contribuido a esta expansión exponencial de la innovación social:

a) El entorno de crisis del estado de bienestar, que marca la reducción de los recursos públicos para la atención a las necesidades sociales tanto de forma pública como a través de las entidades del Tercer Sector, es uno de los principales factores de este protagonismo que, sin duda, ha cobrado la innovación social en los últimos años.

b) El creciente aumento y diversificación de las necesidades sociales y de las personas y grupos que las sufren es otro de los elementos que han impulsado la innovación social.

c) La ineficacia de los modelos preexistentes o al menos el insuficiente impacto de los proyectos, programas y políticas destinados a la reducción de la pobreza, la exclusión y la desigualdad en el mundo.

El contexto actual es un contexto de incertidumbre, de cambios y de enormes desafíos para aquellos que trabajan desde cualquier ámbito en la lucha contra la pobreza y la exclusión y/o por un mundo mejor, más justo y más humano en el que haya igualdad de derechos y oportunidades para todos los seres humanos.

Dado el contexto anteriormente descrito y la certeza de que no va sino a agudizarse en los años venideros, la búsqueda de nuevas soluciones o formas de enfrentar los problemas de la sociedad parece una vía en la que pudiera merecer la pena profundizar.

\section{La Teoría Fundamentada y su aplicación en la investigación}

Este artículo representa la continuidad de la investigación de la tesis doctoral, La innovación social en España: ejes vertebradores desde la Teoría Fundamentada, en la que se pretendía identificar y sistematizar aportaciones de valor para caracterizar, definir y promover la innovación social en España, mediante la metodología de la Teoría Fundamentada.

La Teoría Fundamentada es una metodología que propone un proceso de análisis de los datos para generar teoría sustantiva, proceso que se define a través de recogida y acumulación de datos, su codificación y categorización, la saturación de estas categorías y la relación de éstas entre sí, de forma simultánea.

A continuación, se explican los principios de la Teoría Fundamentada, definiendo los conceptos clave que propone y cómo han sido abordados en esta investigación.

1. Construcción de teoría versus verificación: a diferencia de otras metodologías utilizadas en ciencias sociales, no pone énfasis en verificar teorías preexistentes o demostrar hipótesis preconcebidas, sino en construir y sistematizar la teoría que surge del análisis y la comparación 
constante de los datos. (Glaser \& Strauss, 1967, p. 2).

2. Generación de la teoría como proceso: la generación de una teoría implica un proceso de investigación. Del mismo modo, la generación de una teoría a partir de los datos significa que la mayoría de las hipótesis y conceptos provienen de los datos, sistemáticamente trabajados en el curso de la investigación. «Nuestra estrategia de análisis comparativo para generar teoría pone un gran énfasis en la teoría como proceso, es decir, la teoría como una entidad en constante desarrollo, no como un producto perfeccionado» (Glaser \& Strauss, 1967, p. 32).

3. Importancia de los datos: la teoría debe estar anclada a los datos, proviene de los datos y todos los datos son susceptibles de generar teoría. De acuerdo con Glaser y Straus (1967): «Aunque el énfasis en datos cualitativos es fuerte en nuestro libro, la mayoría de los capítulos también pueden ser utilizados por aquellos que desean generar teoría con datos cuantitativos, ya que el proceso de generación de la teoría es independiente del tipo de los datos utilizados» (p. 18).

4. La acumulación y el análisis de los datos deben hacerse simultáneamente: "Al principio, las hipótesis pueden parecer no relacionadas, pero a medida que las categorías y propiedades emergen, se desarrollan en abstracción y se relacionan, sus interrelaciones acumuladas forman un marco teórico central integrado, el núcleo de la teoría emergente. El núcleo se convierte en una guía teórica para una mayor recopilación y análisis de datos. [...] Cuando la generación de la teoría es el objetivo, sin embargo, uno está constantemente alerta a las perspectivas emergentes que cambiarán y ayudarán a desarrollar su teoría. Estos pueden ocurrir fácilmente incluso el último día de estudio o cuando el manuscrito se revisa en la página de prueba: por lo que la palabra publicada no es la final, sino solo una pausa en el proceso interminable de generación de teoría. Cuando la verificación es el objetivo principal, la publicación del estudio tiende a dar a los lectores la impresión de que esta es la última palabra" (Glaser \& Strauss, 1967, p. 40).

5. La importancia del análisis comparativo y el muestreo teórico: "El muestreo teórico es el proceso de recopilación de datos para generar teoría mediante el cual el analista recopila, codifica y analiza conjuntamente sus datos y decide qué datos se recopilan a continuación y dónde encontrarlos, para desarrollar su teoría a medida que surge. Este proceso de recopilación de datos está controlado por la teoría emergente, ya sea sustantiva o formal" (Glaser \& Strauss, 1967, p. 45).

La Teoría Fundamentada clásica tuvo su origen en un mundo diferente, en 1967, año en que el contexto, los problemas y los medios eran totalmente distintos de lo que tenemos hoy. En la práctica, respecto a la recolección de datos, se hablaba sólo de entrevistas y en contextos muy concretos y localizados.

Hay un hecho fundamental en este cambio sustancial de contexto, la irrupción y normalización de las antes llamadas «Nuevas Tecnologías» que han dejado de llamarse así, para denominarse Tecnologías de la Información y la Comunicación (TIC), pues son asumidas como vehículos comunes de información y comunicación.

Las TIC no sólo han cambiado el contexto en el que se desarrolla el día a día de todos nosotros, sea cual sea nuestra edad y condición, sino que han impactado de forma fundamental en esta investigación ya que, inciden directamente en las formas de recogida y acumulación de datos, en la generación de los datos mismos (tanto en la cantidad, como en la calidad y la velocidad).

Así, el instrumento básico de recogida de datos en tiempos de Glaser y Strauss (1967), los creadores de la Teoría Fundamentada, era la entrevista personal. Ahora, no sólo podemos hacer entrevistas virtuales, sino que los datos primarios se pueden recoger de infinitas fuentes y en tiempo real.

En este sentido, este trabajo ha adaptado la Teoría Fundamentada a un contexto marcado por lo global, con unos datos que tienden a infinito y con unos medios de recolección de datos que van mucho más allá de las entrevistas. Por este motivo, las fuentes de información han sido muy numerosas y diversas y el corpus de investigación es amplio, heterogéneo, representativo y especializado. Consta de más de 
3.000 entradas que han sido codificadas, comparadas, analizadas y saturadas, durante más de 5 años (2013-2018), generando 160 códigos y 26 categorías.

Entre estos 3.000 datos, hay más de 500 ejemplos de innovación social y 50 casos de estudio sobre la innovación social en España, de los cuales 29 son casos de innovación (social) smart.

La aplicación de la metodología de la Teoría Fundamentada clásica adaptada a un contexto 2.0 donde todos somos a la vez receptores y productores de datos, emisores, permite afirmar que hemos hablado de innovación social de una forma radicalmente innovadora y su valor de aportación radica en que otros investigadores pueden seguir este modelo adaptado en futuras investigaciones, en contextos cambiantes y acerca de realidades dinámicas.

\section{Términos y conceptos de base utilizados en el artículo y su definición}

Corresponde ahora definir en este apartado introductorio algunos términos y conceptos que se utilizan en este artículo y sobre los que no existe una definición única, como el término innovación social mismo, o cuyo contenido es punto de partida para este estudio y emerge del propio trabajo de análisis, codificación categorización y comparación constante de los datos, como los retos sociales relevantes (RSR).

\section{a) Innovación social, un concepto en construcción.}

Podría parecer que la innovación social es la suma del significado de dos términos: innovación y social; pero, como ilustraremos con algunos ejemplos, no todo lo que es innovador es social, ni todo lo que es social es innovador.

El 10 de octubre de 2014, al famoso chef Ferran Adrià le conceden el premio a la innovación disruptiva en el South Summit 2014 («Ferran Adrià premio a la Innovación Disruptiva», 2014), un evento que «conecta a los empresarios más innovadores con los inversores más importantes del mundo y las corporaciones que buscan mejorar su competitividad global a través de la innovación» («QQuién está detrás del South Summit?», s.f.), por su creatividad en la cocina y la gastronomía. Es innegable que Adrià es un gran innovador en su campo, la gastronomía, y que ha sido, incluso, rompedor o disruptivo, en el sentido de que ha abierto una brecha en el mercado preexistente y ha creado algo totalmente nuevo. Sin embargo, pese al hecho de ser uno de los precursores de la deconstrucción, descontextualizando este concepto del mundo del arte (consistente en aislar los diversos ingredientes de un plato, generalmente típico, y reconstruirlo de manera inusual, de tal modo que el aspecto y textura sean completamente diferentes mientras que el sabor permanece inalterado), de las espumas (que crea utilizando sifones), de la esferificación (empleo de alginatos para formar pequeñas bolas de contenido líquido), así como el empleo de nitrógeno líquido, etc., no podemos decir que estemos ante un innovador social o que estas aportaciones, a pesar de ser muy innovadoras, supongan innovaciones sociales.

De la misma manera, los comedores sociales para personas sin recursos, a pesar de haber aumentado considerablemente su presencia, número y cantidad de personas asistidas en España durante estos años de crisis, son inminentemente sociales, pero no son innovadores, dado que son medidas asistencialistas que datan del s. XVIII.

Podríamos encontrar numerosos ejemplos en ambos campos, lo innovador y lo social, y no estaríamos hablando de innovación social. Por eso, en realidad, la innovación social no es simplemente la suma de innovación y social, sino que se trata de investigar sobre el lugar en que esos dos conceptos se funden y operan como uno solo.

«La nevera solidaria» es una nevera colocada en la calle, (superando todos los requisitos legales, como el uso de suelo público, requerimientos de sanidad y consumo), para que, aquellas personas a las que les sobre comida preparada, puedan depositarla y aquellas personas que necesiten o quieran un plato de comida casera lo puedan coger. En este caso, estaríamos claramente ante una innovación social. Una innovación social con mayúsculas. Esta es una idea nueva que lucha contra un problema social. Nunca antes se había propuesto una acción tan sencilla como audaz e innovadora, poner una nevera en la calle para luchar localmente contra el desperdicio de comida y contra el hambre de las personas excluidas.

Lo mismo sucede cuando nos referimos a la plataforma de la ONGD Prosalus, \#Yonodesperdicio (s.f.), que permite a particulares que viven o trabajan cerca, regalarse alimentos que por distintas razones (un viaje, un cambio de dieta, una compra por error) no van a consumir. 
Este trabajo recoge, analiza y compara este tipo de ejemplos, donde lo innovador y lo social van intrínsecamente unidos. El hecho de haber realizado la investigación con la metodología de la Teoría Fundamentada tiene como consecuencia que es la propia investigación -el análisis de los datos, su codificación, categorización, saturación de categorías, relación entre categorías y temas, etc. - la que determina la definición de innovación social, su caracterización y su distinción de otros conceptos o fenómenos, a los que he llamado «conceptos o fenómenos colindantes». Naturalmente, existen definiciones de innovación social, pero el debate sobre el concepto no es banal, ya que no existe ninguna definición clara y unívoca.

Como refiere la presentación temática del volumen 36 de la Revista Española del Tercer Sector (2017), dedicado monográficamente a la innovación social, uno de los objetivos básicos que debe perseguir la investigación en este terreno es «aportar claridad conceptual a un fenómeno en auge sobre el que existe un cierto nivel de confusión entre profesionales y académicos» (Mataix Aldeanueva \& Romero Muñoz, 2017, p. 17).

En uno de los trabajos reunidos en ese mismo volumen, «Análisis multidimensional del concepto de innovación social en las organizaciones no lucrativas españolas. Evidencias prácticas» (Álvarez-González, García-Rodríguez, Sanzo-Pérez \& Rey-García, 2017), se recogen varias definiciones de innovación social:... "el término IS se aplica a un conjunto de iniciativas y organizaciones extremadamente heterogéneas, que van desde las intervenciones del tercer sector en su conjunto, a las iniciativas de políticas públicas e incluso hasta las acciones de organizaciones lucrativas que tienen un impacto social marginal" (Borzaga \& Bodini, 2012).

A pesar de esta fragmentación, a la hora de conceptuar la IS se tiende a identificar dos dimensiones básicas: el hecho de que la IS implica un cambio en las relaciones sociales, y que tales cambios intentan resolver un problema socialmente relevante. De esta forma, «cualquier intento de crear una definición general para la IS debe basarse en estos dos componentes comunes, pero diferentes escuelas de pensamiento podrían añadir otras dimensiones» (Van der Have \& Rubalcaba, 2016: 1932), tales como el «empoderamiento o una dimensión tecnológica o económica añadida» (Van der Have \& Rubalcaba, 2016: 1930). (Álvarez-González, García-Rodríguez, Sanzo-Pérez \& Rey-García, 2017, p. 27).
En su publicación Social Innovation. What it is, Why it matters and How it can be accelerated, Mulgan, Tucker y Sanders (2007, p. 8) dicen: "Innovación son nuevas ideas que funcionan"; así, innovación social es entendida como nuevas ideas que funcionan para resolver o gestionar un problema social.

En 2010, la Comisión Europea, en la comunicación titulada Iniciativa emblemática de Europa 2020. Unión por la innovación. Comunicación de la Comisión al Parlamento Europeo, al Consejo, al Comité Económico y Social Europeo y al Comité de las Regiones, define la innovación social como algo deseable, que consiste en:

Encontrar nuevas formas de satisfacer las necesidades sociales que no están adecuadamente cubiertas por el mercado o el sector público (... o) de producir los cambios de comportamiento necesarios para resolver los grandes retos de la sociedad (... y que por tanto) capacitan a los ciudadanos y crean nuevas relaciones sociales y modelos de colaboración. Son, pues, innovadoras por sí mismas y buenas para la capacidad innovadora de la sociedad (Comisión Europea, 2010, pp. 23-24).

Según el informe, La innovación social y las cooperativas: el impacto social de las cooperativas y experiencias socialmente innovadoras: "Enfrentarse a la tarea de establecer un concepto sobre Innovación Social es un trabajo no carente de dificultades. De hecho, si consultamos las últimas investigaciones realizadas por la Comisión Europea, se puede comprobar como en ocasiones las personas investigadoras han rechazado la idea de realizar una definición «estática» del concepto, prefiriendo usar diferentes «nociones» $\mathrm{e}$ interpretaciones, aclarando que el concepto de innovación social no se utiliza para describir o explicar la realidad social sino que «la innovación social debe ser reconocida como un modo particular de acción y de cambio social», definiendo así la Innovación Social como un proceso cambiante y adaptativo al cambio social" (FAECTA, 2014, p. 16).

Efectivamente, en el Informe Social Innovation Research in the European Union Approaches, findings and future directions Policy Review (Jenson \& Harrisson, 2013) se recogen tres definiciones de entre muchas, escogidas por abarcar más aspectos y matices sobre el concepto de innovación social: "Simultáneamente una necesidad social que conduce a nuevas o mejoradas capacidades y relaciones y un mejor uso de los activos y recursos. En otras palabras, las innovaciones sociales son buenas para la 
sociedad y mejoran la capacidad de actuar de la sociedad" (Tepsie).

"La innovación social debe estar estructuralmente orientada a satisfacer la necesidad social (desafío social); Debe implicar un producto, un proceso, un método de comercialización y/o un modelo de organización nuevo o significativamente mejorado" (SELUSI). La innovación social es un proceso en el que los actores de la sociedad civil desarrollan nuevas tecnologías, estrategias, ideas $\mathrm{u}$ organizaciones para satisfacer las necesidades sociales o resolver los problemas sociales (SPREAD). (Jenson \& Harrisson, 2013, p. 15).

Muchos autores consideran necesario hablar de innovación en general antes de hablar de innovación social. En la ya citada ponencia «La innovación social como solución a la crisis: hacia un nuevo paradigma de desarrollo», se señala lo siguiente: "Es obligado hablar de innovación en general como paso previo para referirnos a la innovación social. El concepto de innovación ha venido ganando peso con el paso de los años, convirtiéndose en un concepto fetiche en boga desde la década de los 60s (Rogers, 1962; Nelson $\&$ Phelps, 1966). No obstante, fue Schumpeter quien introdujo la relevancia del concepto y su análisis, al enfatizar que «la innovación es un proceso por medio del cual se introducen en el sistema económico nuevos productos y técnicas» (Schumpeter, 1947). La percepción de Schumpeter sobre la innovación como un proceso de «destrucción creadora» se confinaba al mundo empresarial, al mercado y la tecnología. En todo caso, hemos de reconocer que paralelamente en el tiempo, cuando Schumpeter describía la innovación como un proceso eminentemente económico, Kallen (1949), lo hacía en términos de «cambios culturales o procesos sociales», ampliando su alcance más allá del prisma económico y tecnológico (Hochgerner, 2009). Y el propio devenir económico fue confirmando este hecho.

El informe Meadows en 1972 ya puso los límites al crecimiento exponencial diciendo que el uso de la tecnología, a la que no se oponía, no resolvía los problemas más acuciantes del mundo sino que podía en algún caso intensificarlos y generar efectos colaterales y problemas sociales. Ello puso de manifiesto la necesidad de «otra economía». La Conferencia de Naciones Unidas de Río de Janeiro en 1992 tomó ese testigo al hablar de un desarrollo sostenible desde el punto de vista ecológico, social y económico. Ya más recientemente, se constata que ni la tecnología ni el paradigma de la sociedad industrial son suficientes para alcanzar los Objetivos del
Milenio, sino mediante el estímulo de la innovación social" (ZSI, 2008, p. 28, Fernández Fernández, Montes Pineda \& Asián Chaves, 2012, pp. 1086-1087).

En cualquier texto o documento de los mencionados hasta ahora y prácticamente en cualquier otro material sobre innovación social, se hace referencia a una definición del término innovación social, precisamente por la inexistencia de un concepto unívoco y aceptado por todos.

Al principio de este epígrafe se ha mencionado la aproximación de Mulgan et al., porque podría decirse que son autores, en cierto modo precursores del discurso práctico sobre la innovación social. Estos mismos autores, años después, en la publicación The open book of social innovation (2010) precisan y amplían la anterior definición: "Nuestro interés está en las innovaciones que son sociales tanto en sus fines como en sus medios. Especialmente, definimos las innovaciones sociales como nuevas ideas (productos, servicios y modelos) que a la vez satisfacen las necesidades sociales y crean nuevas relaciones o colaboraciones sociales. En otras palabras, son innovaciones que son buenas para la sociedad y mejoran la capacidad de actuar de la sociedad". (Murray, Caulier-Grice \& Mulgan, 2010, p. 3).

En la Guía de la Innovación Social se define innovación social como: "el desarrollo e implementación de nuevas ideas (productos, servicios y modelos) para satisfacer las necesidades sociales, crear nuevas relaciones sociales y ofrecer mejores resultados. Sirve de respuesta a las demandas sociales que afectan al proceso de interacción social, dirigiéndose a mejorar el bienestar humano" (Comisión Europea, 2013, p. 4).

Además, este informe recoge la definición más concreta de la BEPA (Oficina de Asesores de Política Europea); describe los siguientes tres enfoques clave para la innovación social:

- Innovaciones que respondan a las demandas sociales que tradicionalmente no son dirigidas por el mercado o las instituciones existentes y se dirigen hacia los grupos vulnerables de la sociedad.

- La perspectiva del reto social se centra en innovaciones para la sociedad en su conjunto a través de la integración de lo social, lo económico y lo ambiental.

- El cambio de enfoque sistémico, el más ambicioso de los tres y hasta cierto punto que abarca los otros dos, se logra a través de un proceso de desarrollo 
organizativo cambios en las relaciones entre las instituciones y las partes interesadas. (Comisión Europea, 2013, pp. 4-5).

A base de acumular definiciones de innovación social explícitas o implícitas y de realizar una comparación constante entre las mismas, emerge una teoría anclada en los datos para realizar aportaciones a la definición de innovación social. Aunque la expresión innovación social es frecuentemente utilizada, es conveniente definir los elementos básicos de la misma, la innovación y lo social.

Haciendo una exégesis básica de las dos partes de la expresión, nos referimos, en primer lugar, a innovación, definida en en su acepción más abierta y generosa: no es necesario que se trate de una invención o de una patente; para que sea innovador no hace falta que no hayamos visto u oído nunca algo parecido, basta con que incluya algún elemento novedoso. Obviamente, es inherente a la idea de nuevo o novedoso que sea más eficaz, eficiente o que produzca un impacto mayor. Recordemos aquí la definición ya citada de Mulgan, Tucker y Sanders (2007, p. 8) como «nuevas ideas que funcionan».

Analizando el segundo vocablo de la expresión, social, a continuación vamos a mostrar tres dimensiones necesarias para comprender en toda su profundidad lo que es innovación social. Efectivamente, con la expresión lo social me refiero a tres vectores que deben darse al mismo tiempo para calificar y cualificar a la innovación social. Estas novedades deben, en primer lugar, responder a un reto social relevante o a un problema que importe a la sociedad en su conjunto y cuya solución sea un desafío prioritario para la misma.

La Stanford Graduate School of Business define la innovación social como una «solución novedosa a un problema social, más efectiva, eficiente, sostenible o justa que la solución actual» y que «aporta valor a la sociedad en su conjunto más que a los individuos» («Defining Social Innovation», s.f.). En esta definición empieza ya a aflorar la segunda dimensión de lo social: no sólo tiene que ser bueno para la sociedad, sino también desde la sociedad.

Mulgan et al. (2007) definen innovación social como «actividades y servicios que son motivados por una meta para satisfacer necesidades sociales y que son principalmente desarrolladas y socializadas a través de organizaciones cuyo propósito principal es social» (p. 8).

Phills Jr., Deiglmeier y Miller (2008) hacen una contribución importante al señalar que la innovación social «disuelve fronteras $\mathrm{y}$ promueve un diálogo entre los sectores público, privado y no lucrativo».

Como señalan Murray, Caulier-Grice y Mulgan (2010), la innovación social se define como «nuevas ideas (productos, servicios y modelos) que simultáneamente satisfacen necesidades sociales y crean nuevas relaciones de colaboración. Es decir, que las innovaciones sociales son buenas para la sociedad y aumentan la capacidad de actuar de la sociedad» (Mulgan et al., 2007, p. 3).

Por lo tanto, lo social es tanto en el proceso, el cómo y el porqué de los objetivos sociales y de la sociedad que se desea alcanzar y además fortalece o genera las relaciones entre los distintos agentes sociales: el individuo, la multitud, lo privado y lo público, las organizaciones y movimientos de la sociedad civil y las grandes empresas multinacionales.

Así pues las características inherentes a la innovación social son:

- Nueva, novedosa (y mejor que las precedentes).

- Social de la sociedad.

- Social para la sociedad (hace frente a los RSR para la sociedad).

- Social porque genera nuevas relaciones sociales o fortalece las existentes.

La combinación de estos dos elementos, lo nuevo y lo social, tanto en los medios como en los fines, lleva a una definición de innovación social: Formas nuevas o novedosas que tiene la sociedad, para hacer frente a los retos sociales relevantes (RSR), que son más eficaces, eficientes y sostenibles o que generan mayor impacto que las precedentes y que contribuyen a hacerla más justa, inclusiva, fuerte y articulada.
b) Los retos sociales relevantes: una expresión acuñada durante la investigación.

Al codificar cincuenta casos de innovación social española, la investigación ha permitido desvelar de forma sistematizada cuáles son las preocupaciones de los innovadores sociales en España. Estas preocupaciones, estos desafíos que los innovadores sociales españoles han priorizado en sus innovaciones son los retos sociales relevantes.

El interés de este punto estriba en que, con independencia de las prioridades y objetivos de las distintas agendas a nivel global (Objetivos de Desarrollo Sostenible, Objetivos del Milenio, Indicadores de Desarrollo del PNUD, etc.) o nacional (Objetivos prioritarios de la Cooperación Española, etc.), definidas por los 
estados o por los expertos, los RSR o prioritarios de la innovación española emergen de los propios datos manejados.

Es decir, si en la investigación han aparecido muchos proyectos o casos que se enfrentar al despilfarro de comida, el evitar el despilfarro de comida se convierte en un RSR para la innovación social española.

Los RSR de la innovación social española se pueden agrupar en torno a 5 grandes grupos: 1) Inclusión, 2) Sostenibilidad, 3) Participación e incidencia, 4) Desarrollo y Derechos Humanos y 5) Instrumentales, que a su vez se desglosan en otros, como muestra la Tabla 1.

Tabla1. Retos sociales relevantes de la innovación social española.

\begin{tabular}{|c|c|c|c|c|}
\hline \multicolumn{5}{|c|}{ TEMA: RETOS SOCLALES REIEYANTES DELAINNOVACIÓN SOCLAL ESPANOLLA } \\
\hline INCLUSION & SOSTENIBILDAD & $\begin{array}{l}\text { PARIICIPACIÓN } \\
\text { EINCIDEAICIA }\end{array}$ & $\begin{array}{l}\text { DESARROLLO Y } \\
\text { DDHH }\end{array}$ & INSTRUMENTALES \\
\hline $\begin{array}{l}\text { ACCESIBILIDAD } \\
\text { UNIVERSSAL }\end{array}$ & EMISIONES & ACTIVISMO & ALIMENTACIÓN & COORDINACIÓN \\
\hline DEGÉNERO & ALIMENTOS & CONSUMO & COMERCIO JUSTO & FINANCIACIÓN \\
\hline DEMOCRÁTICA & CONSUMO & DEMOCRACIA & COMUNICACIONES & FORMACIÓN \\
\hline INTERCONFESIONAL & ECOLOGIA & MOBILIZACIÓN & CULTURA & \\
\hline INTERCULTURAL & ENERGIA & & DEMOCRACIA & \\
\hline LABORAL & MEDIOAMBIENTE & & EDUCACIÓN & \\
\hline $\begin{array}{l}\text { PERSONAS CON } \\
\text { DISCAPACIDAD / } \\
\text { ENFERMEDAD } \\
\end{array}$ & POBLACIÓN & & ENERGIA & \\
\hline SOCLAL & RECICLANE & & JUSTICIA & \\
\hline \multirow[t]{3}{*}{ TECNOLÓGICA } & \begin{tabular}{|l} 
RECURSOS \\
NATURALES \\
\end{tabular} & & SALUD & \\
\hline & & & TRANSPORTE & \\
\hline & & & VIVIENDA & \\
\hline
\end{tabular}

Fuente: Herrero de Egaña, 2018a, p. 385

Como se explica a continuación, que hagan frente al menos a uno de los RSR es, a su vez, uno de los criterios de selección de los casos de estudio.

\section{Los casos de estudio: criterios de selección}

Para realizar el presente estudio se han seleccionado cincuenta casos de una masa total de más de 600, 300 de ellos españoles, atendiendo al carácter representativo de los mismos, de forma que, a partir de su análisis cualitativo y de las realidades que representan, pudiera generalizar rasgos característicos de la innovación social en España y quizá atisbar factores que la favorezcan e impulsen. Para determinar dicho carácter representativo de los casos, se ha utilizado cuatro criterios selectivos, a saber:

1. Reto social relevante: Para que un caso sea seleccionado, es necesario que la innovación social haga frente a un reto o problema social relevante, real y prioritario. Para determinar qué y cuáles son los RSR, se han asumido que han revelado la propia investigación, es decir, aquellos a los que los actores de la innovación social dan mayor importancia, a la luz de las innovaciones sociales que surgen para hacerles frente o para solucionarlos. Así pues, si la investigación ha revelado como reto social relevante la inclusión de las personas con discapacidad, se han buscado y seleccionado aquellos casos que facilitan dicha inclusión.

2. Elemento(s) innovador(es): Este criterio supone que serán potencialmente incluidos aquellos casos que contengan uno o varios elementos innovadores, que los diferencien de otras iniciativas similares en cuanto al problema enfrentado o en cuanto a la solución propuesta. Para determinar cuáles son los elementos innovadores, al igual que para determinar los RSR, de nuevo son los que se han evidenciado por la propia investigación, y que radican en torno a tres ejes: el modo, el sujeto y el ámbito.

- El modo es la categoría que agrupa códigos que tienen que ver con una nueva forma de hacer las cosas (de forma colaborativa, disruptiva, smart, entre otras), de generar los cambios sociales; tienen que ver con el cómo.

- El ámbito es la categoría que agrupa códigos que se refieren a nuevos ámbitos en los que actúa la innovación social, más allá de las fronteras geográficas tradicionales (glocal, virtual, acupuntura urbana, etc.); tiene que ver con el dónde.

- El sujeto: esta categoría agrupa una serie de códigos referidos a nuevos actores sociales (la multitud, los prosumidores, etc.); tiene que ver con el quién.

Estos nuevos actores, nuevos ámbitos y nuevos modos de hacer, son los elementos innovadores que nos pueden llevar a realizar una clasificación de las innovaciones sociales en función del elemento o elementos innovadores que contengan.

3. Cambio que generan: Este criterio implica que se han seleccionado casos que generan un cambio en la realidad social en la que quiere incidir, cuyos resultados sean objetivamente verificables y cuenten con indicadores de impacto. En el ya citado artículo «Análisis multidimensional del concepto de innovación social en las organizaciones no lucrativas españolas. Evidencias prácticas», se afirma: "Creemos necesario incluir, a la hora de 
definir la IS, esta dimensión adicional basada en los resultados de la misma desde el punto de vista de la «mejora explícita» en términos de eficiencia, eficacia y sostenibilidad" (ÁlvarezGonzález, García-Rodríguez, SanzoPérez \& Rey-García, 2017).

4. Lugar en que se produce la innovación: Este criterio prioriza casos en España.

\section{Innovación Social, Innovación Smart}

Del presente trabajo podría deducirse que nos referimos a la misma cosa cuando hablamos de innovación social y de innovación smart, sin embargo es necesario hacer una aclaración respecto a esta afirmación.

Generalmente se ha venido asociando el concepto smart a la ciudad:

"Las Smart City como ciudades que aplican la limitación de recursos, la sensibilidad por la eficiencia en su uso y la adecuada gestión de los vectores ambientales que determinan el buen funcionamiento de las ciudades, tienen el objetivo de proveerla de una infraestructura que intente garantizar un desarrollo sostenible, un incremento de la calidad de vida de sus ciudadanos, una mayor eficiencia de sus recursos humanos y energéticos. Resumidamente, se trata de usar la tecnología en beneficio de la población. Para que una Smart City triunfe, tiene que superar el concepto ciudad como organización administrativa, y convertirse en Territorio Inteligente que venga de la mano de determinadas características ambientales, sociales, económicas, culturales donde se ponga de manifiesto la importancia de la ciudadanía. Una ciudad puede abordarse desde ámbitos donde la ciudadanía no tenga concurso ninguno, o bien desde un prisma de territorio urbano con una perspectiva que incluya a la población del mismo" (MiCiudadRECI, s.f.).

En España existen en la actualidad ochenta y dos ciudades inteligentes, todas ellas miembros de la RECI (Red Española de Ciudades Inteligentes), cuyo objetivo es: "intercambiar experiencias y trabajar conjuntamente para desarrollar un modelo de gestión sostenible y mejorar la calidad de vida de los ciudadanos, incidiendo en aspectos como el ahorro energético, la movilidad sostenible, la Administración electrónica, la atención a las personas o la seguridad” («Quienes somos», 2017). Sin embargo, se entiende smart, inteligente, como una sociedad que invierte en servicios públicos de alta calidad, en la productividad, competitividad e innovación de sus servicios y empresas, en el emprendimiento, en la formación y capacitación de las personas, en la participación e inclusión de sus ciudadanos y en la sostenibilidad. Si bien es verdad que generalmente se utiliza este concepto refiriéndose a un entorno urbano, smart city o ciudad inteligente, la investigación ha evidenciado una concepción de smart que va más allá del entorno urbano: ser smart es una actitud que se puede mantener en un entorno rural.

Así pues, aunque hay muchos elementos comunes entre la innovación social en general y la innovación (social) smart, cuando hablamos de casos de innovación smart o inteligente en España, en esta investigación, hablamos de veintinueve casos de estudio que además de ser considerados como innovación social, son innovaciones smart, y no siempre, como veremos a continuación, se desarrollan en entornos urbanos.

\subsection{La Innovación Smart en España}

En el trabajo original a partir del cual se inspira este artículo, se establece que los elementos innovadores radican en torno a tres ejes: el modo, el sujeto y el ámbito, como nos hemos referido anteriormente.

Estos nuevos actores, nuevos ámbitos $\mathrm{y}$ nuevos modos de hacer, son los elementos innovadores que nos pueden llevar a realizar una clasificación de las innovaciones sociales en función del elemento innovador dominante.

Hablamos de elemento innovador dominante porque tal y como se observa en la figura 1, un alto porcentaje de los casos de innovación social analizados (un $82 \%$ ) son innovadores tanto en el modo como en el sujeto y el ámbito, y un $92 \%$ son innovadores tanto en el modo como en el ámbito. El $100 \%$ de los casos son innovadores en el modo.

Se puede afirmar, por tanto, que las innovaciones sociales lo son por más de un motivo o en más de un aspecto.

El grupo más numeroso de elementos innovadores es el que se enmarca dentro de la categoría modo, que se refiere a cómo se hacen las cosas (Figura 1). Es decir, en innovación social las cosas se hace siempre de otro modo.

El modo smart es el segundo modo más innovador de hacer las cosas (Figura 2). 
Figura 1. Elementos innovadores en los 50 casos de estudio.

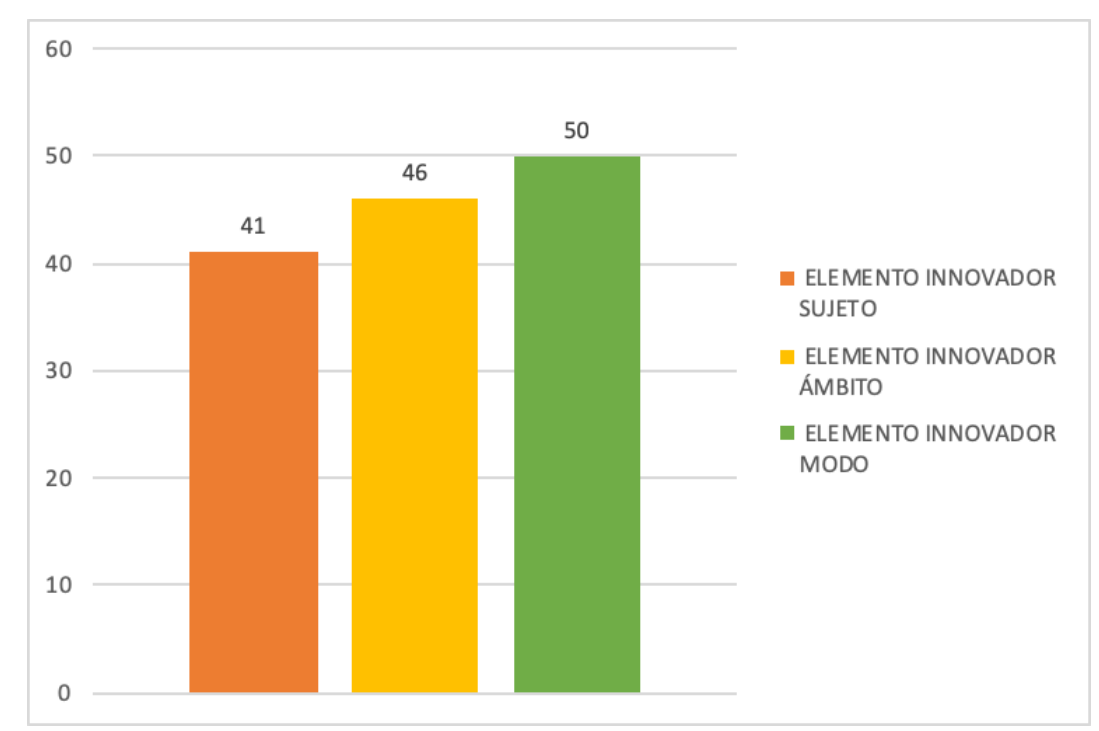

Fuente: Elaboración propia.

Figura 2. Modos de ser innovador en los 50 casos de estudio.

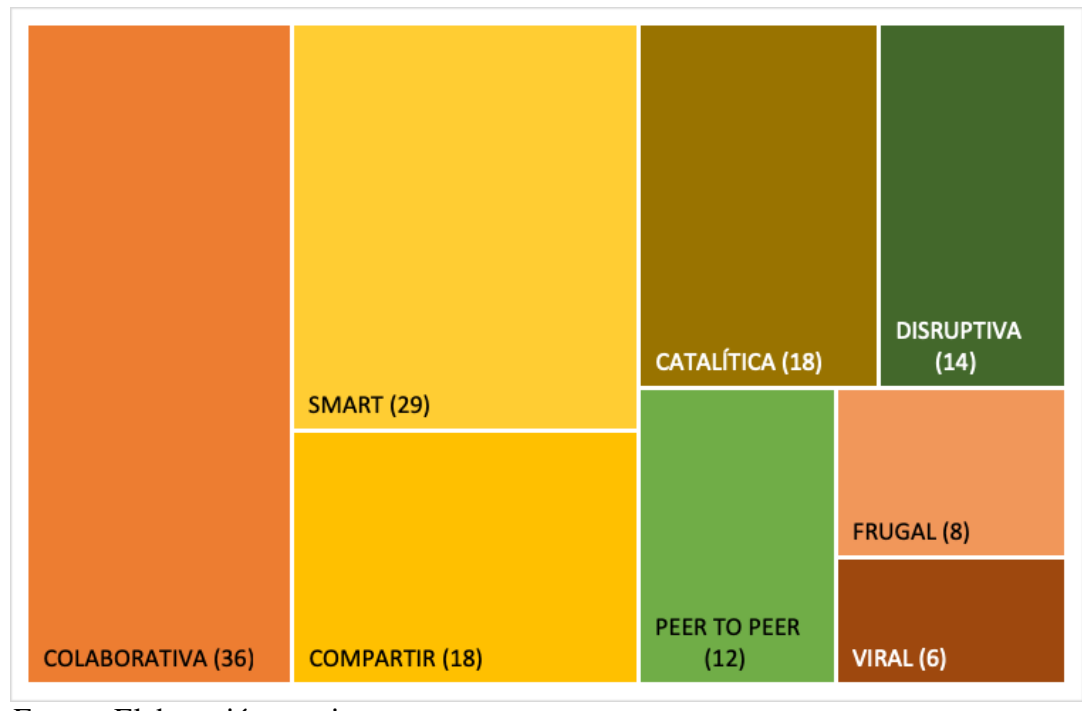

Fuente: Elaboración propia.

Tal y como se observa en la Figura 2, veintinueve de los cincuenta casos de estudio contienen una innovación en el modo que hemos caracterizado como smart, lo que representa un $58 \%$ del total de los casos de innovación social analizados.

A partir de este momento el análisis se centra en los veintinueve casos de innovación social española que han sido clasificados específicamente como smart.

\section{2.Ámbito de la Innovación Social Smart en España}

En primer lugar, debemos señalar que, si bien la innovación social inteligente en España se da principalmente en ámbitos urbanos, también hay un creciente número de casos en entornos eminentemente rurales o mixtos, como refleja la Figura 3.

La distribución de las innovaciones sociales smart en España, por provincias la podemos ver en la Figura 4. 
Figura 3. Distribución de las innovaciones smart en relación a su ámbito.

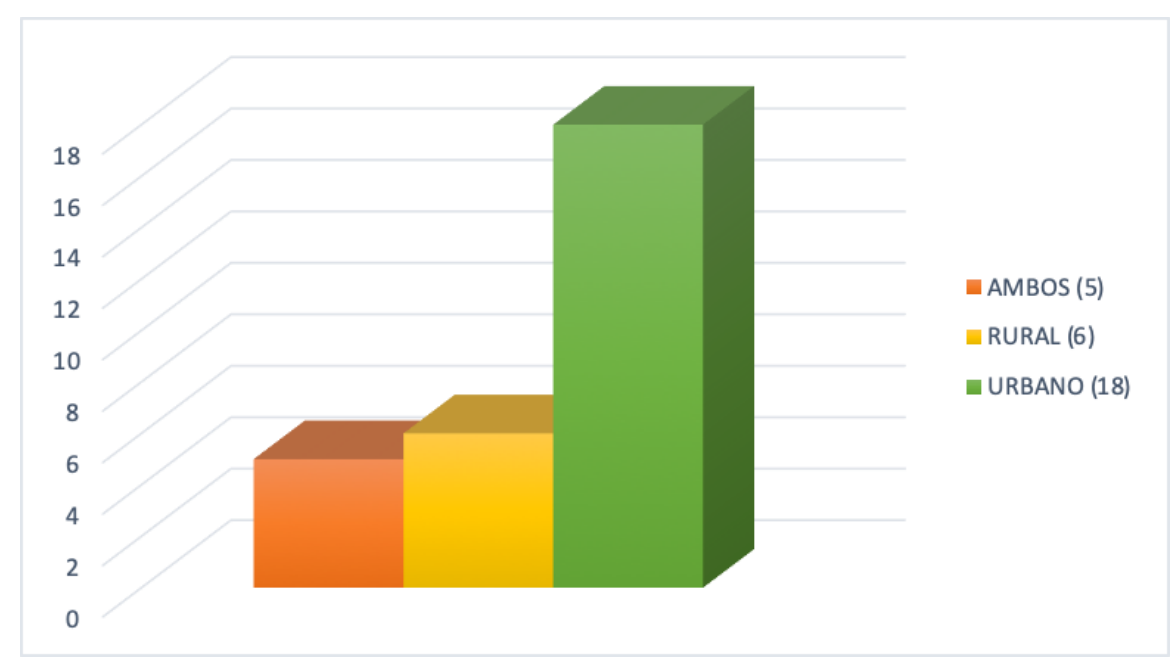

Fuente: Elaboración propia.

Figura 4. Distribución de las innovaciones smart en relación a su ámbito y provincia.

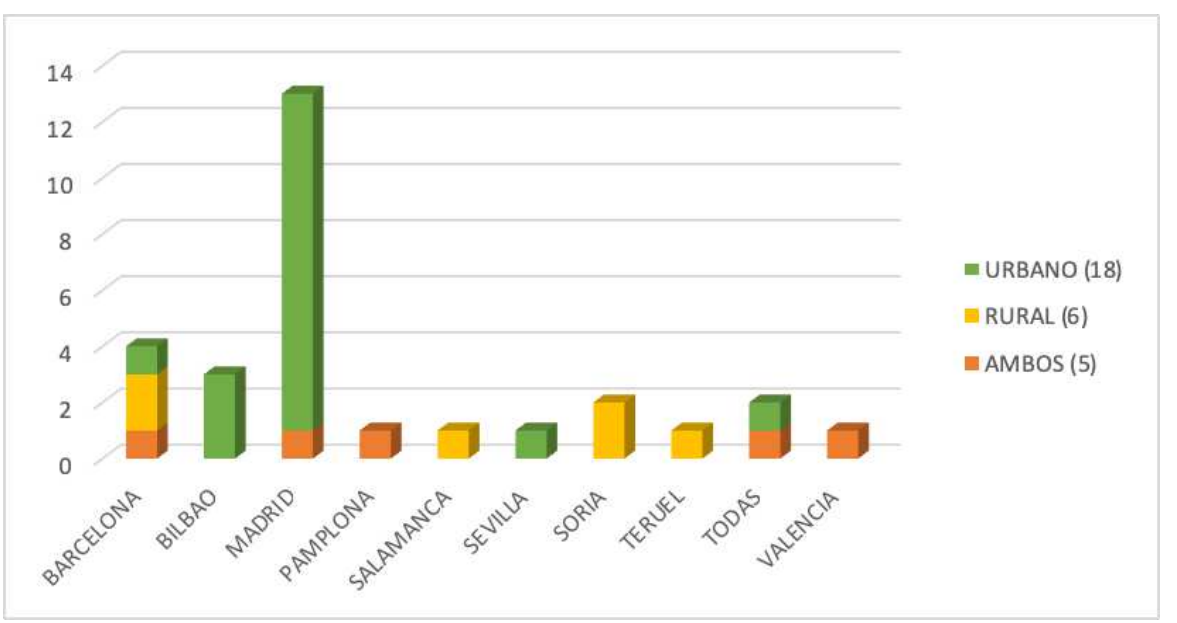

Fuente: Elaboración propia.

\subsection{Sujeto de la innovación social Smart en España}

En cuanto al tipo de sujeto que lidera las innovaciones sociales smart objeto de este estudio, llama la atención la escasa representación del sector público frente al sector privado, si bien hay algunas iniciativas de tipo mixto, como se puede observar en la Figura 5.

\subsection{Otros elementos innovadores de la innovación social smart en España}

Recordemos que el elemento smart es un elemento de innovación relativo al modo, recordemos también que en la innovación social española un alto porcentaje de los casos de estudio tienen elementos innovadores en cuanto al modo, y, al mismo tiempo, al sujeto $\mathrm{y}$ al ámbito.

Pero, ¿cuál es la relación entre lo smart o inteligente y los elementos innovadores relativos al ámbito y al sujeto?

Como se refleja en las Figuras 6 y 7, las innovaciones sociales smart innovan en el sujeto en un $83 \%$ y en el ámbito en un $90 \%$, lo que quiere decir que al menos veintiséis casos de innovación social smart son innovadores en el ámbito y el sujeto. 
Figura 5. Distribución de las innovaciones smart en relación al tipo de sujeto.

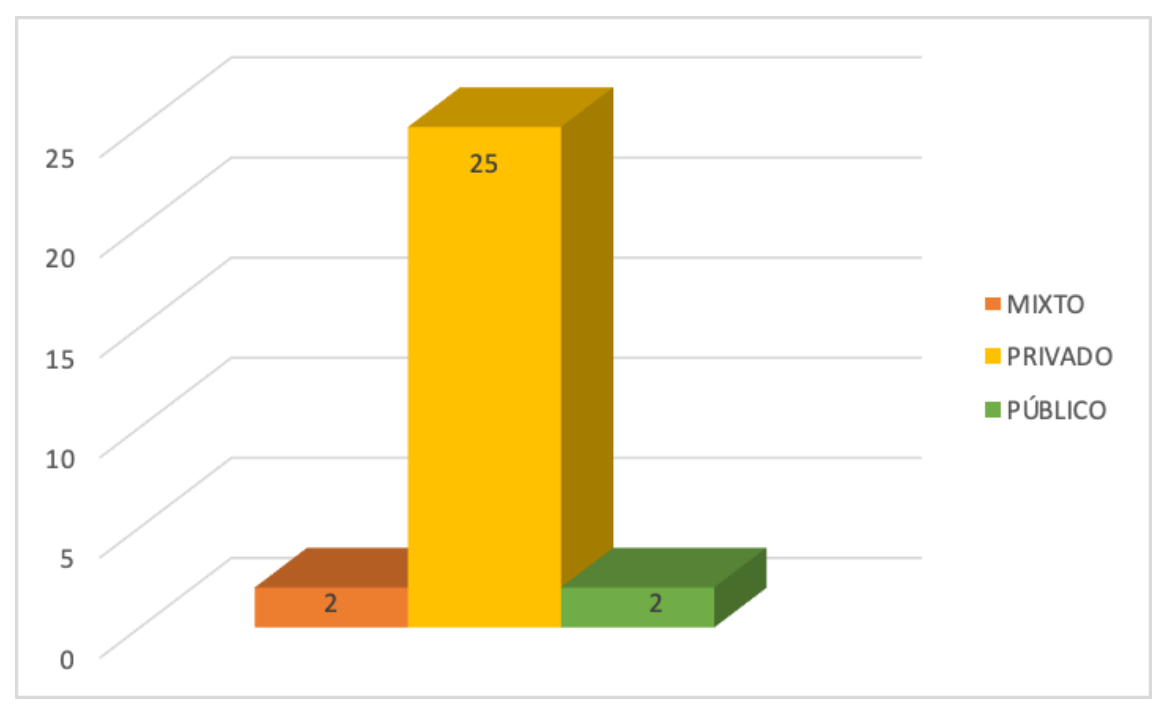

Fuente: Elaboración propia.

Figura 6. Distribución de las innovaciones smart en relación a los sujetos innovadores.

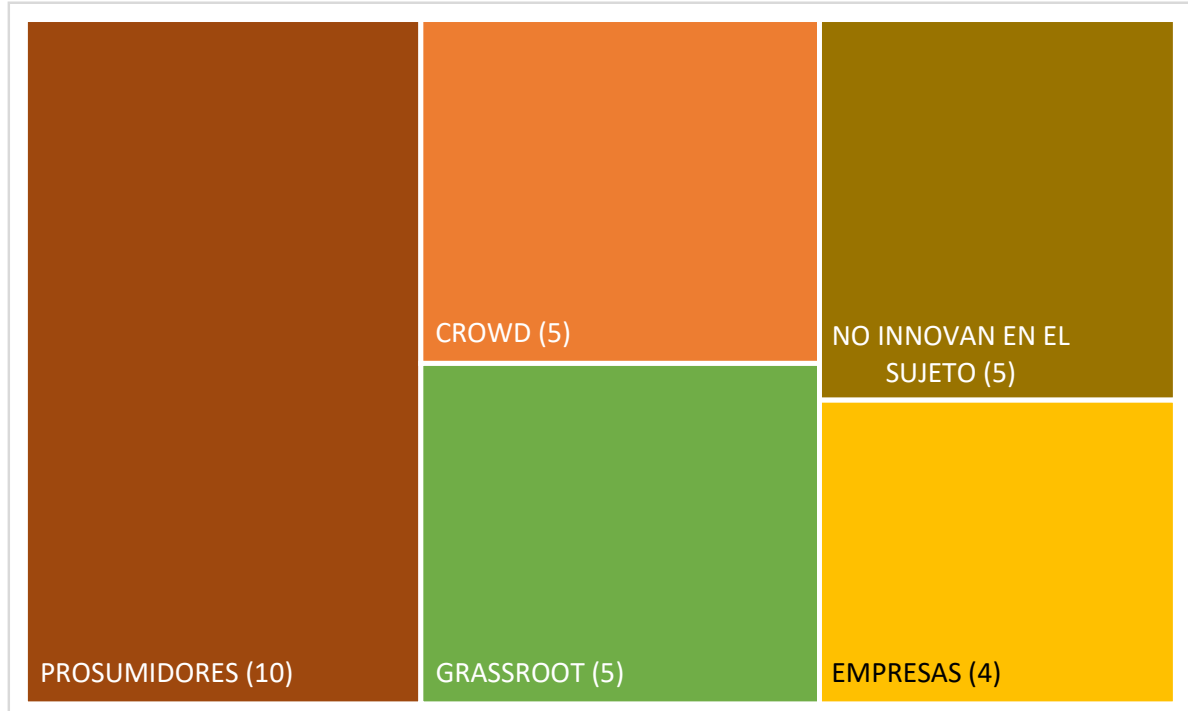

Fuente: Elaboración propia.

En la figura anterior se observa que los sujetos innovadores mayoritarios de la innovación social Smart son (1) los prosumidores, fusión de las palabras productor (en inglés, producer y productor) y consumidor (consumer) que se refiere al consumidor que, con su comportamiento consciente y su capacidad de evaluar los pros y los contras de distintos tipos bienes o servicios, genera una información acerca de tendencias emergentes (generalmente en los medios 2.0, aunque también por medios tradicionales), que las empresas y otras organizaciones deben incorporar, de forma que se convierte en una especie de consumidor/productor; (2) la multitud o crowd, la gente, un grupo indefinido de gente, una multitud que actúa y promueve una innovación social, y (3) la gente común o grassroots, que entendemos como la asociación natural y espontánea, desde la base de los miembros de un grupo o comunidad, con fines de apoyo comunitario o grupal, donde la comunidad o el grupo actúa por iniciativa propia en un entorno específico. Suman entre los tres un $69 \%$, información que podemos conectar con la que se recoge en la Figura 5. De los 29 casos de innovación social smart, 25 están promovidos 
por un actor privado y en 24 de ellos este actor privado es además, un nuevo actor.

Figura 7. Distribución de las innovaciones smart en relación a los ámbitos innovadores.

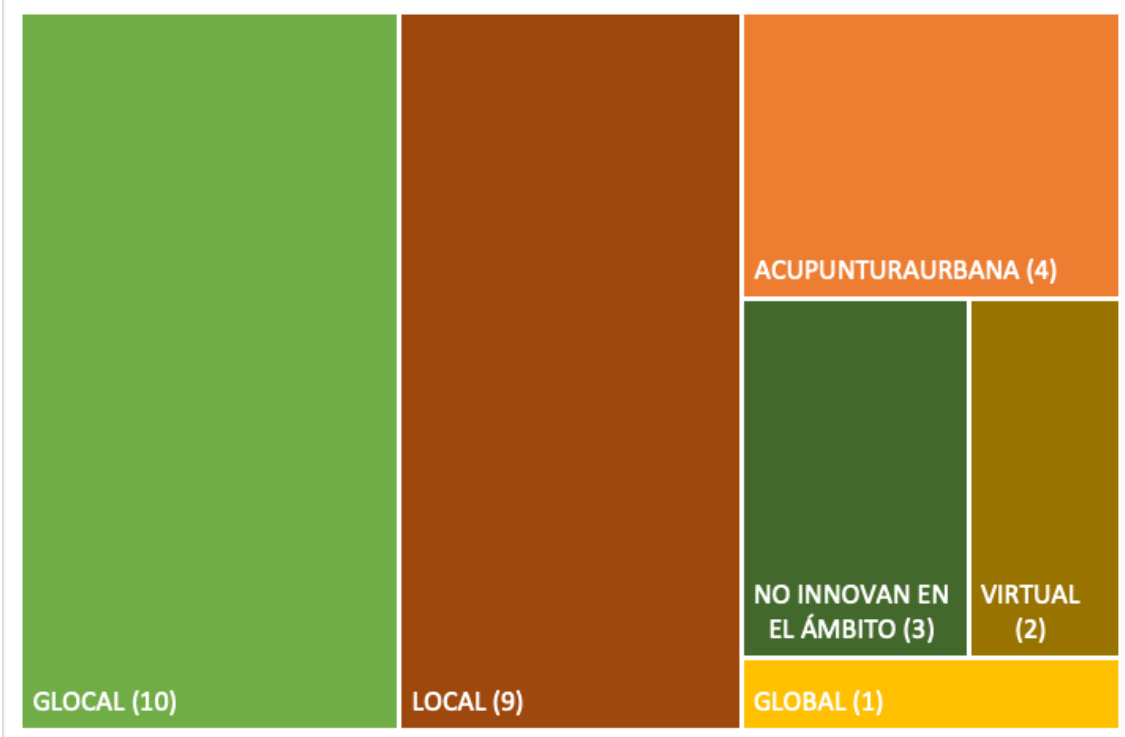

Fuente: Elaboración propia.

Como puede deducirse de la figura anterior, los ámbitos innovadores mayoritarios de la innovación social smart son (1) lo glocal, es decir, el ámbito que mezcla los elementos locales y particulares con los globales y que está representado por la frase «pensar globalmente y actuar localmente»; (2) lo local, y (3) la acupuntura urbana, entendida como la intervención urbana, puntual, local, pequeña y sutil, hecha desde abajo, que concibe la ciudad como un ser vivo y que genera un impacto positivo que se expande por toda la ciudad, al igual que en la acupuntura china las agujas liberan la energía y revitalizan el todo mediante la curación de las partes. Son menos relevantes los ámbitos global y virtual, hecho que resulta lógico, dados los fines de la innovación social smart.

\subsection{Retos sociales relevantes de la innovación social smart en España}

A lo largo de este texto nos hemos referido en numerosas ocasiones a los retos sociales relevantes de la innovación social española.
Es importante señalar que esta es una locución acuñada durante la investigación que se refiere a los desafíos que los innovadores sociales españoles han priorizado en sus innovaciones. En las Figuras 8 y 9 se puede apreciar cómo varían los RSR si hablamos de innovación social en España, en general o si hablamos de innovación social smart, ya que los RSR referidos a la sostenibilidad y a la incidencia son muy superiores en la innovación social smart.

En la ponencia «Retos sociales relevantes de la innovación social española y grandes retos europeos: análisis comparado basado en el estudio de casos», presentada en 2018 en el XVII Congreso Internacional de Investigadores en Economía Social y Cooperativa CIRIEC (Herrero de Egaña, 2018b), ya quedó de manifiesto la alineación en términos generales de los RSR de la innovación social española y los desafíos que plantean distintas agendas. En dicha ponencia se debatió ampliamente la conexión entre los ODM, los ODS y los RSR y se pudo evidenciar hasta qué punto la innovación social española impacta positivamente en los logros y desafíos europeos y globales. 
Figura 8. RSR de la innovación social española.

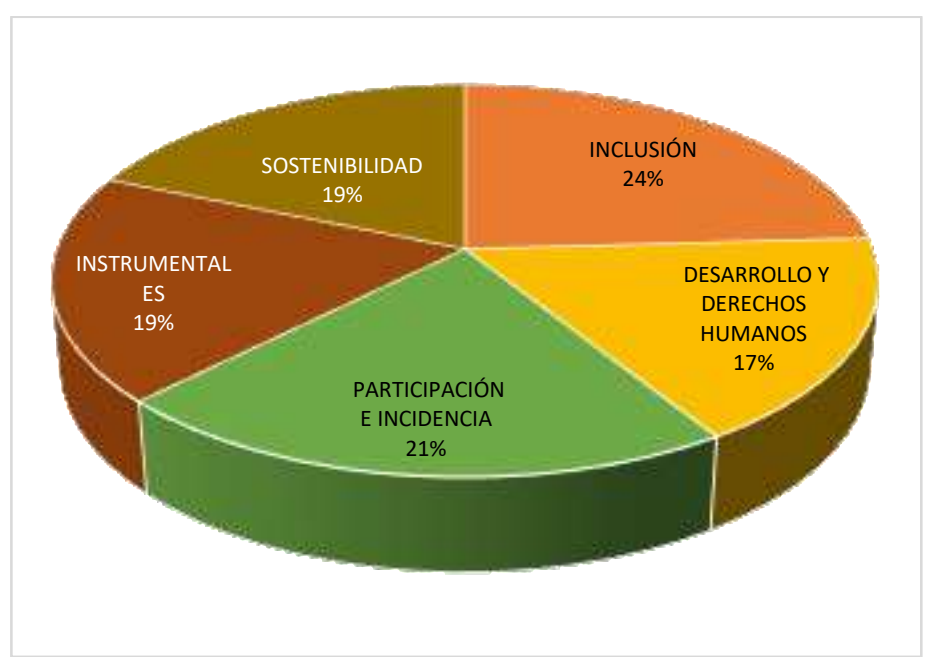

Fuente: Elaboración propia.

Figura 9. RSR de las innovaciones smart.

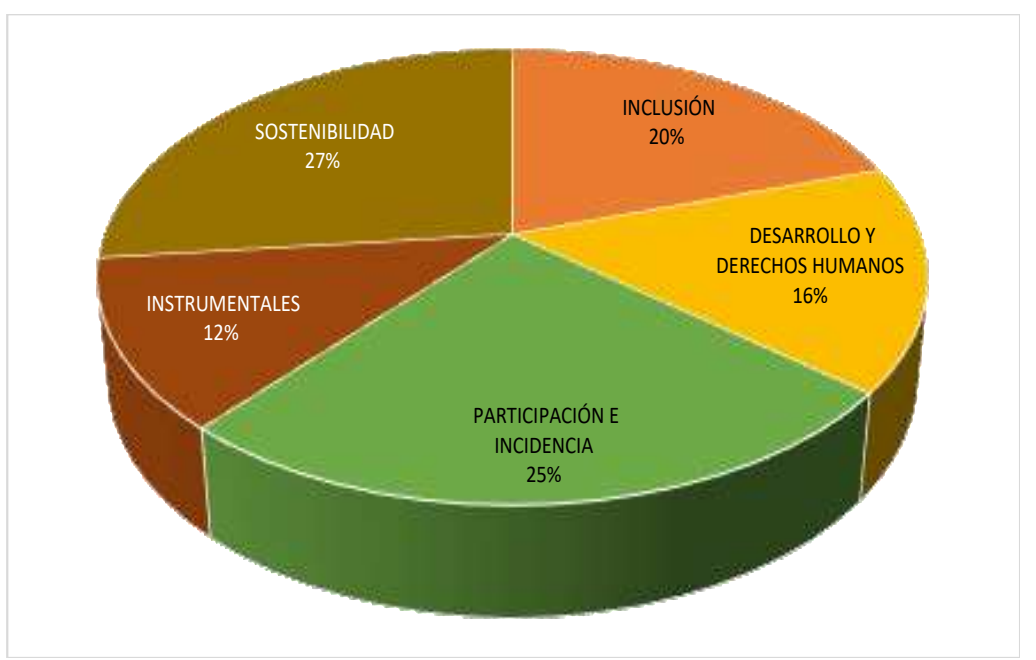

Fuente: Elaboración propia.

\section{Conclusión}

Hablamos de innovación inteligente o smart cuando representa una solución buena para la comunidad y sostenible con el medio ambiente en un entorno social.

Así pues, la innovación social en cierta medida smart y la innovación

smart en cierta medida es social.

La innovación social en general y la innovación social smart contribuyen a hacer frente a los desafíos de la humanidad y, por tanto, a un mundo mejor, más justo y más humano. Efectivamente, hemos podido evidenciar que los retos sociales relevantes o desafíos que se han marcado como prioritarios los innovadores smart en España, contribuyen y se alinean en un alto grado con los ODM y los ODS, agendas supranacionales. Así pues, podemos afirmar que la innovación smart española contribuye significativamente a una agenda global por el desarrollo integral, humano y sostenible.

Algún día no será necesario hacer esta diferenciación y todas las caracterizaciones de la innovación serán redundantes ya que no habrá innovación si no es social y no habrá innovación social si no es smart. 


\section{Referencias}

Álvarez-González, L. I., García-Rodríguez, N., Sanzo-Pérez, M. J. \& Rey-García, M. (2017). Análisis multidimensional del concepto de innovación social en las organizaciones no lucrativas españolas. Revista Española del Tercer Sector, 36, 27-48. Recuperado de: https://www.accioncontraelhambre.org/sites/default/files/documents/revista_rets_n36.pdf

Comisión Europea. (2010). Iniciativa emblemática de Europa 2020. Unión por la innovación. Comunicación de la Comisión al Parlamento Europeo, al Consejo, al Comité Económico y Social Europeo y al Comité de las Regiones. Bruselas: Unión Europea. Recuperado de: http://www.idi.mineco.gob.es/stfls/MICINN/Internacional/FICHEROS/Actuaciones_Europeas/Comuni cacion_Union_por_la_Innovacion.pdf

Comisión Europea. (2013). Guía de la Innovación Social. Comisión Europea. http://www.hisenda.gva.es/documents/599445/599488/Traducci\%C3\%B3n+de+la+Gu\%C3\%ADa+de+ Innovaci\%C3\%B3n+Social.pdf/86278f94-5f81-4df7-b949-61e69c583588

Defining Social Innovation. (s.f.). Stanford Graduate School of Business. En línea. Recuperado de: https://www.gsb.stanford.edu/faculty-research/centers-initiatives/csi/defining-social-innovation

FAECTA (en colaboración con Grupo Cooperativo Gekos) [= Pino Merlo, R. D., Jurado, L. M., Comet Herrera, D., Fernández, M. J., Sánchez Bárcena, G. \& Kalas Porras, G. G.]. (2014). La Innovación Social y las Cooperativas: el impacto social de las cooperativas y experiencias innovadoras socialmente. FAECTA. Recuperado de:

https://www.faecta.coop/fileadmin/documentos/PDF_FAECTA/faecta_Informe_INNOVACION_SOCI AL_DEFINITIVO.pdf (Acceso enero 01, 2015).

Fernández Fernández, M. ${ }^{a}$ T., Montes Pineda, O. \& Asián Chaves, R. (2012). La innovación social como solución a la crisis: hacia un nuevo paradigma de desarrollo. Los costes de la crisis y alternativas en construcción. XIII Jornadas de Economía Crítica, Sevilla: Universidad de Sevilla, 1084-1101.

Ferran Adrià premio a la Innovación Disruptiva. (Octubre, 2014). Luisannet Arte y Tecnología. Recuperado de: http://www.luisan.net/blog/marketing/ferran-adria-recibio-premio-la-innovacion-disruptiva

Glaser, B. G. \& Strauss, A. L. (1967 [2006]). The Discovery of Grounded Theory Strategies for Qualitative Research. New Brunswick - Londres: Aldine Transaction.

Herrero de Egaña, B. (2018a). La innovación social en España: ejes vertebradores desde la Teoría Fundamentada. Tesis doctoral de la Universidad de Comillas. Madrid.

Herrero de Egaña, B. (2018b). Retos sociales relevantes de la innovación social española y grandes retos europeos: análisis comparado basado en el estudio de casos. XVII Congreso Internacional de Investigadores en Economía Social y Cooperativa CIRIEC, Toledo, 4-5 de octubre de 2018. Toledo: CIRIEC España - Universidad de Castilla La Mancha, 26 pp.

Jenson J. \& Harrisson D. (2013). Social innovation research in the European Union Approaches, findings and future directions. Policy Review. Luxemburgo: Publications Office of the European Union. Recuperado de: http://www.net4society.eu/_media/social_innovation.pdf

Mataix Aldeanueva, C. \& Romero Muñoz, S. (2017). Presentación temática. Revista Española del Tercer Sector, 36, 17-19. Recuperado de: https://www.accioncontraelhambre.org/sites/default/files/documents/revista_rets_n36.pdf

MiCiudadRECI (s.f.). Mi Ciudad Inteligente: Análisis y difusión de las Smart Cities en España. http://www.miciudadinteligente.info/mi-ciudad-inteligente/

Mulgan, G., Tucker, S., Ali, R. \& Sanders, B. (2007). Social Innovation. What it is, why it matters and how it can be accelerated. Oxford: Said Business School. Recuperado de: http://youngfoundation.org/wpcontent/uploads/2012/10/Social-Innovation-what-it-is-why-it-matters-how-it-can-be-accelerated-March2007.pdf 
Murray, R., Caulier-Grice, J. \& Mulgan, G. (2010). The Open Book of Social Innovation. London: The Young Foundation / Nesta. Recuperado de : http://youngfoundation.org/wp-content/uploads/2012/10/TheOpen-Book-of-Social-Innovationg.pdf

Phills Jr., J. A., Deiglmeier, K. \& Miller, D. T. (2008). Rediscovering Social Innovation. Stanford Social

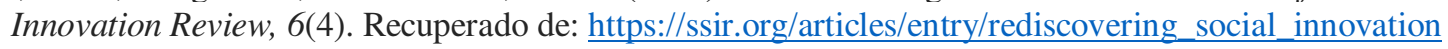

Quienes somos. (2017). Smartcity. Recuperado de: http://www.redciudadesinteligentes.es/index.php/sobre-la$\underline{\mathrm{red} / q u i e n e s-s o m o s}$

¿Quién está detrás del South Summit? (s.f.). South Summit. Recuperado de: https://es.southsummit.co/about

Yonodesperdicio (s.f.). [Plataforma para impedir el desperdicio de alimentos]. Yonodesperdicio. Recuperado de: https://yonodesperdicio.org/page/faqs?locale=es 